\title{
Flow of Gas-Liquid Foam through Constrictions
}

\author{
Mandar Badve, Mostafa Barigou \\ School of Chemical Engineering, University of Birmingham \\ Edgbaston, Birmingham B15 2TT, United Kingdom \\ M.P.Badve@bham.ac.uk; M.Barigou@bham.ac.uk
}

\section{Extended Abstract}

The movement of gas-liquid foams through narrow complex passages is commercially challenging and technically important. Gas-liquid foams enjoy numerous industrial applications including food, consumer goods, oil recovery, foamed concrete, firefighting and mineral processing. They are complex structured fluids with non-linear viscoelastic characteristics, and fundamental understanding of their properties and flow dynamics is therefore limited. Specifically, flow though constrictions can have detrimental effects on foam structure and rheology. Such flows are crucial in many industrial processes, e.g. flow and moulding of aerated products such as ice cream and molten chocolate, foam sclerotherapy in treatment of vascular diseases or pumping and deployment of foam cement slurries in narrow annuli. Thus, a foam with inadequate rheological properties may not readily flow through narrow passages and constrictions, giving poor 'fill'.

In this paper, we report on a study of the flow of gas-liquid foam through two-dimensional narrow channels containing sudden or gradual contractions and expansions. The ability to observe foam structure and stability during flow is essential, and having 2D transparent channels allows foam flow to be observed systematically in complex geometries. Foams with formulations of varying degrees of complexity (interfacial properties, liquid rheology) are studied. Thus, a full analysis of foam flow and rheology through 2D channels with constrictions is obtained and related to foam structure, physical properties, and any instabilities or perturbations observed therein. Foam behaviour is examined as a function of flowrate, initial bubble size and flow constriction geometry. The effects on the entire flow field including foam morphology, liquid holdup, pressure drop and velocity profile are elucidated. We examine individual bubble motion and deformation using strain rates and a "texture tensor". In this way, we explore the parameter space to obtain the local strain and stress fields and determine transition from elastic to plastic deformation. 